\title{
A computer-controlled administration of the matching familiar figures test
}

\author{
ANDREW O. HUMMEL-SCHLUGER and JOHN S. BAER \\ University of Washington, Seattle, Washington
}

\begin{abstract}
The Kagan Matching Familiar Figures Test of Impulsivity/Reflectivity was modified to allow computer control of stimulus presentation and data acquisition via IBM-compatible systems. The comparability of computer administration and traditional hand administration was examined in a splithalf study with a sample of 58 students (ages, 18-39 years). Analyses of variance revealed no significant differences between the administration techniques for latency and a small but significant difference for accuracy. Correlations between the two administration techniques were .61 for latency and .40 for error scores. Internal consistency coefficients for computer administration exceeded those for hand administration on both latency and accuracy. These data compare favorably with results reported by others and suggest that computer administration offers a viable alternative to traditional hand administration of the MFFT in research applications.
\end{abstract}

In the present investigation, we assess the reliability of a DOS-based program for computer administration of the Matching Familiar Figures Test (MFFT). Developed by Kagan, Rosman, Day, Albert, and Phillips (1964), the MFFT has been used to examine the construct of impulsivity/reflectivity and its relationship to a variety of other cognitive and behavioral characteristics. The MFFT consists of series of visual tasks, each of which presents the subject with a standard figure and a set of variants. Only one of the variant figures represents a precise match to the standard. The remainder differ from the standard in one or more details. Subjects are required to choose the matching figure and are rated according to the speed and accuracy of their responses. Although the MFFT has its critics (Gerdje, Block, \& Block, 1985; Milich \& Kramer, 1984 ) and indeed is not considered to be a valid diagnostic measure of impulsivity, it remains one of the primary instruments used in research to investigate cognitive dimensions of reflectivity and impulse control. The visual nature of the task, as well as the need to assess speed (or latency) of responding, makes the MFFT an excellent candidate for computer administration.

The adolescent/adult version of the MFFT consists of 14 items. Two of these are used for practice and are not scored. The traditional method of administration involves presenting the prototype and the eight response figures on two facing pages of a flip book. The experimenter records the accuracy of the subject's responses and the latency to the subject's first response.

This research was supported by Grant 5-RO1-AA08632 from the National Institute for Alcohol Abuse and Alcoholism. The authors wish to thank Allison Wilkens for assistance in the design and execution of this study, and Jennifer Watson and Kristine Luce for helpful guidance in the preparation of the manuscript. Requests for reprints and computer source code may be sent to A. Hummel-Schluger, Department of Psychology, Mailstop JF-18, University of Washington, Seattle, WA 98105 (e-mail: ahummel@u.washington.edu).
Like many hand-administered instruments, the MFFT is susceptible to unwanted variability, ranging from experimenter-induced performance anxiety to deterioration of materials. Experimenters must be trained and available. Administration of such instruments by computer provides a more uniform testing environment. Van Merriënboer and colleagues have conducted two studies to examine the reliability of the MFFT under computercontrolled administration. In one, Van Merriënboer and Jelsma (1988) reported test-retest correlations of .78 and .74 for latency and accuracy data between hand and computer-assisted administration via a DOS-based microcomputer that controlled advancement from one item to the next. Presentation of items was, however, still accomplished through the use of a flipbook, and the computer's role was limited to giving an auditory cue to turn to the next item and to collecting the typed responses. In a second study, Van Merriënboer, Jelsma, Timmermans, and Sikken (1989), examining a fully automated version of the MFFT for the Apple Macintosh, found test-retest correlations of .68 and .66 (hand-tocomputer and computer-to-hand, respectively) for latency data, but .36 and .78 for accuracy data. A similar fully automated version of the MFFT for DOS-based systems would be of great benefit to researchers using IBM-compatible microcomputers. Here we present such a version, along with preliminary normative data on young adults.

\section{METHOD}

\section{Program Development}

For this study, each of the 14 items was digitized and converted to a bitmapped graphics file for display on an IBM-compatible microcomputer. A program (MFFT-PC) was written in Borland Turbo Pascal to control the presentation of items and collect response times and accuracy data. The items were displayed as black line drawings on a white background on a 14-in. VGA monitor at a resolution of $640 \times 480$ pixels. Monitor limitations necessitated 
Table 1

Total Latency by Trial, Administration Technique, and Set Number

\begin{tabular}{|c|c|c|c|c|c|c|c|c|}
\hline \multirow{3}{*}{$\begin{array}{c}\text { Administration } \\
\text { Technique }\end{array}$} & \multicolumn{4}{|c|}{ Trial 1} & \multicolumn{4}{|c|}{ Trial 2} \\
\hline & \multicolumn{2}{|c|}{ Set 1} & \multicolumn{2}{|c|}{ Set 2} & \multicolumn{2}{|c|}{ Set 1} & \multicolumn{2}{|c|}{ Set 2} \\
\hline & Lat. & $S D$ & Lat. & $S D$ & Lat. & $S D$ & Lat. & $S D$ \\
\hline Hand & 229.60 & 112.11 & 184.86 & 92.19 & 247.60 & 160.27 & 243.14 & 93.25 \\
\hline Computer & 201.21 & 111.98 & 158.43 & 84.89 & 233.99 & 124.97 & 253.43 & 132.11 \\
\hline
\end{tabular}

a reduction in size of all items with respect to their printed versions. The average amount of reduction was $43.7 \%(S D=10.9)$.

\section{Subjects}

The sample consisted of 58 ( 31 male, 27 female) undergraduate students enrolled in introductory psychology classes at the University of Washington. The subjects' age averaged 21.1 years $(S D=$ 4.1). Extra course credit was awarded to participating students.

\section{Design}

For the reliability study, the 14 individual items of the MFFT were separated into two sets of 7 items, the odd-numbered items constituting Set 1 and the even-numbered items constituting Set 2. Each set consisted of one "practice" item and six "real" items. The subjects were randomly assigned to four groups (1-4) that differed in terms of the order in which the sets were presented and which set was computer administered. This design provided counterbalancing to account for effects due to order of administration and potential differences in difficulty between the two sets. Hence, each group received both sets of MFFT items: one set was administered manually and the other by computer. Groups 1 and 3 received Set 1 by hand and Set 2 by computer, whereas Groups 2 and 4 received Set 2 by hand and Set 1 by computer. Groups 1 and 2 received hand administration in Trial 1 , followed by computer administration for Trial 2. The reverse was true for Groups 3 and 4

\section{Procedure}

Subjects were tested individually while seated comfortably in a quiet room with sufficient space to accommodate both hand and computer administration. The computer was located on a movable table that could be rolled in front of the subject. Prior to each administration, subjects were instructed in the task. Instructions were presented orally before hand administration and visually before computer administration.

Hand administration was performed through use of a flip book. Subjects indicated their selection by pointing. The experimenter recorded accuracy and the latency to the first response with a handheld stopwatch. The MFFT-PC required the subjects to indicate their responses on the screen with the use of a trackball-controlled pointing system. The computer recorded response latencies and accuracy. Owing to concern that subjects might feel less pressured by the absence of a stopwatch under computer administration, the following sentence was added to the instruction set: "The computer will be keeping track of how long it takes you to find the matching figure."

Under both administration techniques, if the initial response was incorrect, the subject was instructed to choose again. Latency for subsequent responses was not recorded. The subject proceeded to the next item if the correct response was given or if the subject made eight consecutive incorrect responses. The total number of incorrect responses was recorded under both administrations.

\section{RESULTS AND DISCUSSION}

An analysis of variance (ANOVA) with a repeated measure of trial number ( 1 or 2 ) and between-subjects factors of administration technique (hand or computer first) and item set ( 1 or 2 first) was performed on total latency. The data are displayed in Table 1 . The analysis yielded no significant effect of administration technique $[F(1,54)=1.49$, n.s. $]$ or item set $[F(1,54)=0.05$, n.s. $]$. An effect of trial number was found: Irrespective of administration technique and item set, subjects responded more quickly on the set of items presented first $[F(1,54)=$ $18.36, p<.001]$. No significant interactions were found between any of the factors.

Table 2 displays mean latency to first response for each item under both MFFT and MFFT-PC methodologies. As can be seen, there is considerable similarity in item latency across the two methods of administration. Univariate between-subjects comparisons of means showed no significant differences between the two administration techniques.

A similar ANOVA of accuracy produced similar results. These data are presented in Table 3 . A significant effect of trial number $[F(1,54)=4.98, p=.030]$ indicated the consistent finding that subjects missed more items in the first set that they received. The analysis also revealed a significant effect of administration technique on total items missed $[F(1,54)=4.32, p=.042]$ with computer administration subject to a higher error rate $(3.35 / 6$ items; $2.95 / 6$ items for hand administration). Item set $[F(1,54)=$ 0.88, n.s.] was shown to be nonsignificant.

Correlations between the two administration techniques were significant for both latency $(r=.61, p<$ $.001)$ and accuracy $(r=.40, p=.001)$. Similarly, there were strong correlations between latency and accuracy under both hand administration $(r=-.49, p<.001)$ and computer administration $(r=-.72, p<.001)$. Analysis of internal consistency among latency scores revealed

Table 2

Latencies to Initial Response Hand Versus Computer Administration

\begin{tabular}{lccccccc}
\hline & \multicolumn{2}{c}{ Hand } & & \multicolumn{2}{c}{ Computer } \\
\cline { 2 - 3 } & Lat. & $S D$ & & Lat. & $S D$ & $F$ & $p$ \\
\hline Dog & 25.33 & 16.61 & & 31.22 & 17.29 & 1.70 & .20 \\
Rose & 31.43 & 21.24 & & 37.32 & 24.65 & 1.03 & .32 \\
Soldier & 45.90 & 29.29 & & 41.08 & 26.95 & 0.41 & .53 \\
Graph & 33.96 & 16.93 & & 32.97 & 18.55 & 0.05 & .83 \\
Baby & 52.47 & 42.08 & & 46.93 & 30.05 & 0.32 & .57 \\
Lamp & 24.64 & 11.21 & & 23.57 & 13.82 & 0.11 & .74 \\
Dress & 45.03 & 33.16 & & 38.93 & 26.41 & 0.58 & .45 \\
Lion & 52.93 & 35.44 & & 45.27 & 38.69 & 0.67 & .41 \\
Glasses & 28.73 & 13.94 & & 27.06 & 16.34 & 0.19 & .67 \\
Plane & 41.14 & 23.32 & & 39.68 & 28.52 & 0.05 & .83 \\
Leaf & 41.13 & 21.52 & & 32.37 & 20.83 & 2.48 & .12 \\
Bed & 29.89 & 11.56 & 27.12 & 16.15 & 0.55 & .46 \\
\hline
\end{tabular}


Table 3

Overall Error Rate (E) by Trial, Administration Technique, and Set Number

\begin{tabular}{|c|c|c|c|c|c|c|c|c|}
\hline \multirow{3}{*}{$\begin{array}{l}\text { Administration } \\
\text { Technique }\end{array}$} & \multicolumn{4}{|c|}{ Trial 1} & \multicolumn{4}{|c|}{ Trial 2} \\
\hline & \multicolumn{2}{|c|}{ Set 1} & \multicolumn{2}{|c|}{ Set 2} & \multicolumn{2}{|c|}{ Set 1} & \multicolumn{2}{|c|}{ Set 2} \\
\hline & $\mathrm{E}$ & $S D$ & $E$ & $S D$ & $\mathrm{E}$ & $S D$ & $\mathrm{E}$ & $S D$ \\
\hline Hand & 3.07 & 1.33 & 2.86 & 1.35 & 2.93 & 1.33 & 2.93 & 1.44 \\
\hline Computer & 3.21 & 1.25 & 4.27 & 1.03 & 3.00 & 1.47 & 2.87 & 1.55 \\
\hline
\end{tabular}

Cronbach's alphas of .91 (Set 1) and .89 (Set 2) for computer administration and .90 (Set 1) and .83 (Set 2) for hand administration. Accuracy data revealed alphas of .33 (Set 1) and .50 (Set 2) for computer administration and .24 (Set 1) and .37 (Set 2) for hand administration. Messer (1976), in his review of studies of impulsivity/ reflectivity cited reliability coefficients of .89 for latency and .58 and .62 for accuracy. Although the accuracy coefficients obtained in the present study may seem low by comparison, it must be recalled that Cronbach's alpha is a function of the number of items as well as their intercorrelation. The reported alphas in this study reflect reliabilities within 6-item sets in contrast to the standard 12. (Computation of a reliability coefficient across all 12 items, but which does not compare administration techniques, reveals an alpha of .54. This compares favorably with reliabilities reported in the literature.)

The varied reduction in size of items for the MFFT-PC did not generate significant effects upon the data. Item analysis comparing percentage of reduction from MFFT to MFFT-PC showed no significant correlation with latency, accuracy, or change in either.

While the latency data reported here are consistent with (if somewhat lower than) those reported by Heckel, Hiers, Laval, and Allen (1980) for hand administration on undergraduate students, the accuracy data are not. Heckel et al. (1980) report a 12 -item error rate of 3.25 ; the extrapolated 12-item error rate would be 5.89 in the present study. It is likely that these differences are caused by differing experimental procedure. The procedure reported by Heckel et al. (1980) allowed for only a single response; the present study allowed subjects to change their response up to eight times. This may well have resulted in a different solving strategy among the present subjects by emphasizing the ability to reevaluate an untenable hypothesis. Van Merriënboer et al. (1989), using the same procedure as that in the present study, reported an average of 5.5 errors.

The differences observed between the two administration techniques in total number of items missed may also be due to unforeseen differences in experimental procedure. Although the experimenter remained in the room during the MFFT-PC, the computer collected each subject's data and provided feedback, and the experi- menter was not an active participant in the test. This may have reduced the negative impact of an incorrect response by lessening perceived performance anxiety and thus allowed a more "free-wheeling" approach.

Van Merriënboer et al. suggested in 1989 that results showing greater latencies and fewer errors under computer administration may have been due to a novelty effect in subjects with little or no computer experience. Although no data were collected to measure computer familiarity, the present study, with subjects who were young children when the first IBM PC was marketed, suggests that perhaps that novelty has worn off.

In summary, we believe computer administration to be a viable alternative to the traditional method of presenting the Matching Familiar Figures Test. At this early stage of development, further normative data must be collected before its use in a clinical or diagnostic setting can be proposed. Generalization from the present data should, of course, be made cautiously, owing to the population that was sampled: college students at an average age of 21 . Assessment of other age groups and clinical populations must await further testing. Nevertheless, few mean differences were noted between computer and hand administration, and the reliability estimates of latency scores were remarkably high. Research applications relating MFFT performance to other measures of interest can reap immediate benefits from several advantages: ease of use, reduction of experimenter-induced variability, and the ability to incorporate it into a battery of other computer-administered tasks.

\section{REFERENCES}

GERDJE, P. E., BLock, J., \& Block, J. H. (1985). Longitudinal consistency of matching familiar figures test performance from early childhood to preadolescence. Developmental Psychology, 21, 262-271.

Heckel, R. V., Hiers, J. M., Laval, C. J., \& Allen, S. S. (1980). Adult norms on the Kagan Matching Familiar Figures Test of Impulsivity/ Reflectivity. Catalog of Selected Documents in Psychology, 11, 5.

Kagan, J., Rosman, B. L., Day, D., Albert, J., \& Phillips, W. (1964). Information processing in the child: Significance of analytic and reflective attitudes. Psychological Monographs, 78(1, Whole No. 578).

Messer, S. B. (1976). Reflection-impulsivity: A review. Psychological Bulletin, 83, 1026-1052.

Milich, R., \& KRAMER, J. (1984). Reflections on impulsivity: An empirical investigation of impulsivity as a construct. Advances in Learning \& Behavioral Disabilities, 3, 57-94.

Van Merriënboer, J. J. G., \& Jelsma, O. (1988). The Matching Familiar Figures Test: Computer or experimenter controlled administration? Educational \& Psychological Measurement, 21, 181-186.

Van Merriënboer, J. J. G., Jelsma, O., Timmermans, J., \& Sikken, J. (1989). Computerized vs. experimenter controlled administration of the Matching Familiar Figures Test: Mean test scores and reliabilities. Educational \& Psychological Measurement, 49, 883-891.

(Manuscript received October 24, 1994; revision accepted for publication January 24, 1995.) 